\title{
Prevención primaria del suicidio y el Puente Chilina de Arequipa.
}

\author{
Primary prevention of suicide and the Chilina Bridge of Arequipa. \\ Ángela Villantoy-Echegaray a, Lizardo Cruzado ${ }^{1,2, b}$
}

\section{Sres. Editores:}

La palabra prevención proviene del latín "praeventious" (prae: antes; eventious: acontecimiento), y literalmente significa "lo que ocurre antes" o "lo que se anticipa". Este concepto es piedra angular en la salud pública y medicina preventiva, por cuanto la prevención constituye el acto de impedir que algo suceda. Clásicamente, la prevención se ha estructurado en tres niveles: la prevención primaria, que evita por completo que se produzca la enfermedad porque elimina sus causas; la prevención secundaria, que detecta precozmente la enfermedad cuando es asintomática aún y un tratamiento precoz puede detener su progresión, y finalmente la prevención terciaria, que se refiere a las actividades clínicas que previenen un deterioro adicional o disminuyen las complicaciones después de que una enfermedad se ha declarado por sí misma (1).

Usualmente se concibe al suicidio como el hecho terminal de una cadena que se inicia con los deseos de morir, seguidos por los deseos y planes suicidas, luego por intentos de mayor o menor severidad y que desembocan en el suicidio consumado. Es decir, el suicidio como un evento premeditado es el modelo imperante en la valoración del fenómeno suicida (en realidad, este prototipo es vigente sobre todo para los trastornos depresivos) (2). Sin embargo, existe creciente evidencia de que muchas veces el suicidio se desarrolla con mínima planificación: a esto se denomina "suicidio impulsivo", término que ya había empleado Durkheim allá por 1897 (2). Aunque no existe una definición estandarizada, se incluye como suicidio impulsivo a aquellos en los que entre la ideación y la ejecución del acto median pocos minutos a pocas horas (no hay base empírica para estos puntos de corte). La mayoría de estudios encuentra que los suicidios impulsivos constituyen más de la mitad de todos los intentos de suicidio (3).

Aunque no existe una meridiana división entre las características de los suicidas impulsivos y los no impulsivos, es un hecho que ante un acto suicida sin mayor premeditación, resultará crucial evitar que el acceso a los medios más letales resulte fácil. La Organización Mundial de la Salud reconoce explícitamente que restringir el acceso a medios altamente letales (plaguicidas muy tóxicos como órganofosforados, armas de fuego, saltos desde altura) es eficaz para la prevención primaria del suicidio pues así los potenciales suicidas tienen más tiempo disponible de reflexión y para buscar opciones alternativas (4).

La eficacia de estas medidas ha sido sustentada tanto teórica como empíricamente. En el caso de los suicidios por defenestración, existe evidencia de que tras la construcción de barreras elevadas en el Viaducto Príncipe Eduardo (Ontario, Canadá),el segundo puente con mayor número de suicidios registrados en el mundo, los suicidios se redujeron a cero (5). En el caso del Golden Gate, el puente con mayor número de suicidios en el mundo, los estudios costo / beneficio (6) y las evidencias de estudios de prevención en lugares semejantes (7) han llevado a

\footnotetext{
${ }^{1}$ Instituto Nacional de Salud Mental "Honorio Delgado - Hideyo Noguchi”. Lima, Perú.

${ }^{2}$ Universidad Peruana Cayetano Heredia. Lima, Perú.

${ }^{a}$ Médica cirujana

${ }^{\mathrm{b}}$ Médico psiquiatra
} 


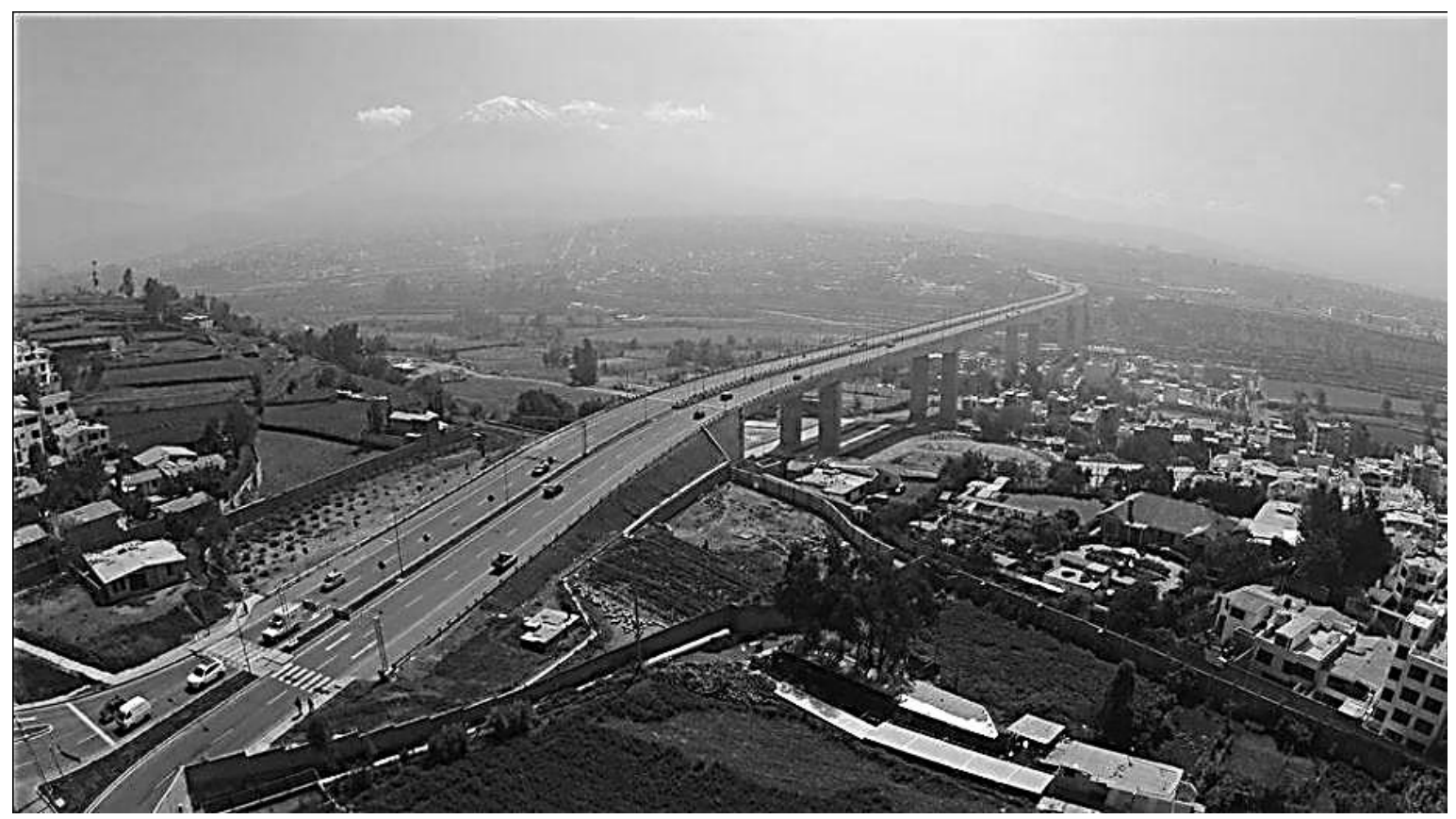

Figura 1. Puente Chilina de Arequipa (Fuente: Wikimedia Commons).

que este año se instalen finalmente mallas protectoras a los costados del puente. Y lo más importante, luego de las medidas de prevención, los suicidios no se incrementaron en áreas cercanas como podría pensarse: que al cercar un puente, el intentador de suicidio fácilmente reemplazará la alternativa bloqueada por otra, por lo que supuestamente resultaría inútil esa forma de prevención (7). Inclusive en un seguimiento a los suicidas frustros del Golden Gate, se halló que posteriormente no más de $10 \%$ conseguían el suicidio (8). La frase "el suicida está dispuesto a morir" representa esta creencia estigmatizante pues en realidad la gran mayoría de suicidas se halla ambivalente entre la vida y la muerte, si logran superar la crisis pueden recuperar su deseo de vivir.

A nivel local también hay evidencia de la exitosa prevención primaria del suicidio: el Puente Villena de Miraflores fue escenario de 120 suicidios consumados a partir de su apertura en 1968; desde el año 2004, en que se colocaron las vallas elevadas a este puente, no se ha consumado ningún suicidio desde su altura de 80 metros (9).

La ciudad de Arequipa, a 1000 kilómetros al sur del Perú, posee el puente urbano más extenso del Perú: el Puente Chilina, de 562 metros de longitud y 40 metros de altura (figura 1); desde su inauguración en el año 2014 hasta julio de este año 2018, 19 personas se han quitado la vida lanzándose desde su plataforma (10).
Los intentos de suicidio han sido aún más numerosos y el escaso personal de serenazgo asignado para su vigilancia difícilmente puede custodiar el extenso puente, hasta la fecha, no se ha acordado la colocación de vallas que impidan el lanzamiento de personas que buscan el suicidio. Acápite aparte sería ahondar en el efecto de la prensa en la difusión de las noticias sobre suicidios y su influencia en el incremento del número de estos casos.

El suicidio es un fenómeno multifactorial y enormemente complejo: tal vez no siempre podrá lograrse satisfactoriamente su prevención pero a los que tenemos una buena oportunidad de salvar son a aquellas personas que, en su momento, tienen éxito en su primer intento debido a los métodos letales que han elegido (4). Es lamentable que el Puente Chilina de Arequipa siga siendo una puerta abierta a suicidios impulsivos. Las instancias respectivas de la salud mental y la psiquiatría, desde el campo gubernamental y profesional, debieran pronunciarse.

\section{REFERENCIAS BIBLIOGRÁFICAS}

1. Bloom M, Gullota TP. Definitions of primary prevention. In: Gullota TP, Bloom M (eds.) Encyclopedia of Primary Prevention and Health Promotion. $2^{\text {nd }}$ ed. New York: Springer; 2014. pp. 3-12.

2. Rimkeviciene J, O'Gorman J, De Leo D. Impulsive 
suicide attempts: A systematic literature review of definitions, characteristics and risk factors. J Affect Disord. 2015; 171:93-104.

3. May AM, Klonsky ED. "Impulsive" suicide attempts: What do we really mean? Personal Disord. 2016;7(3):293-302.

4. Organización Mundial de la Salud. Prevención del suicidio: un imperativo global. WashingtonDC: Organización Panamericana de la Salud; 2014.

5. Sinyor M, Schaffer A, Redelmeier DA, Kiss A, Nishikawa Y, Cheung AH, et al. Did the suicide barrier work after all? Revisiting the Bloor Viaduct natural experiment and its impact on suicide rates in Toronto. BMJ Open. 2017;7: e015299. doi:10.1136/ bmjopen-2016-015299

6. Atkins D, Woods DL. Analysis of the cost effectiveness of a suicide barrier on the Golden Gate Bridge. Crisis. 2013; 34(2):98-106.
7. Pirkis J, Too LS, Spittal MJ, Krysinska K, Robinson J, Cheung YT. Interventions to reduce suicides at suicide hotspots: a systematic review and metaanalysis. Lancet Psychiatry. 2015;2(11):994-1001.

8. Seiden RH. Where Are They Now? A follow-up study of suicide attempters from the Golden Gate Bridge. Suicide Life Threat Behav. 1978; 8(4): 203216.

9. Muñoz J, Vega-Dienstmaier J, Mendoza C, Muñoz H. Suicidio e intento de suicidio por salto de altura en el puente Villena. Rev Neuropsiquiatr. 2005; 68 (34):140-152.

10. La República. Nuevo suicidio en Puente Chilina de Arequipa, suman 19. Lima: La República; 23 de julio del 2018. (Fecha de acceso Diciembre del 2018) Disponible en: en: https://larepublica.pe/ sociedad/1283810-arequipa-nuevo-suicidio-puentechilina-suman-19 